\title{
Intraperitoneal Administration of Lactobacillus casei Prolongs the Life Span of AKR Mice
}

\author{
Takashi Watanabe and Hajime Saito* \\ Department of Microbiology and Immunology, Shimane Medical University, \\ Izumo, 693 Japan
}

(Received 22 April, 1987)

\begin{abstract}
Intraperitoneal administration of heat-killed Lactobacillus casei YIT 9018 (LC 9018) markedly prolonged the life span and inhibited the enlargement of the thymus of 2and 3-month-old female AKR/J mice, but not those of 5-month-old female mice.
\end{abstract}

Key words : Lactobacillus case $i$; AKR mice; life span

LC 9018, a lyophilized preparation of heat-killed Lactobacillus casei YIT 9018, has a potent antitumor activity against various allogeneic and syngeneic tumor cells in experimental animals $(3,13)$. The antitumor activity may be due to the stimulation of host-immune mechanisms, in terms of activation of macrophages (4), natural killer cells (5) and antibody-dependent killer cells (13). On the other hand, it was reported that $L$. case $i$ among the 10 species of the genus Lactobacillus showed long-term duration of protective action against Pseudomonas aeruginosa and Listeria monocytogenes infections in mice, as a result of the activation of murine macrophages due to the intraperitoneal and intravenous administration of the agent (9, 10). L. casei also exhibited strong protective action against herpes simplex virus infection in mice (12). However, it is unclear whether or not $L$. case $i$ is also effective against spontaneously occurring tumors.

AKR mice are characterized by high incidence of spontaneous recombinant murine leukemia viruses (MuLVs) between ecotropic and xenotropic (mink cell focusinducing) MuLV-sequences $(2,7)$ which had been integrated near into the $c-m y c$ gene locus, as a proviral gene insertion (6).
This paper deals with the effect of LC 9018 on the life span of female AKR/J mice.

LC 9018 was donated by Yakult Central Institute for Microbiological Research, Tokyo, Japan. Five-week-old female and male $\mathrm{AKR} / \mathrm{J}$ mice were purchased from Seiwa Experimental Animals Co., Fukuoka, Japan. The neonatal mice were maintained in an air-conditioned clean room. Ten each of 2-, 3-, or 5-month-old female AKR mice were given intraperitoneally (ip) $0.1 \mathrm{ml}$ of $2 \mathrm{mg} / \mathrm{ml}$ of LC 9018 suspended in saline twice weekly for 8 weeks. As controls, mice $(n=10)$ were injected ip with $0.1 \mathrm{ml}$ of saline, under the same conditions. The body weight and the number of survivors were recorded once weekly for 52 to 54 weeks. On the other hand, the 2-month-old female mice given LC 9018 were decaptitated 12 weeks after the last injection, and the thymus removed and weighed.

Figure 1 shows the cumulative mortality rates of LC 9018-administrated and control 2-month-old female AKR mice. Deaths in control and experimental groups first occurred at 20 and 32 weeks after birth, respectively, and all the mice died at 38 weeks in the former group and at 54 weeks in the latter group. Mean survival ages in control 


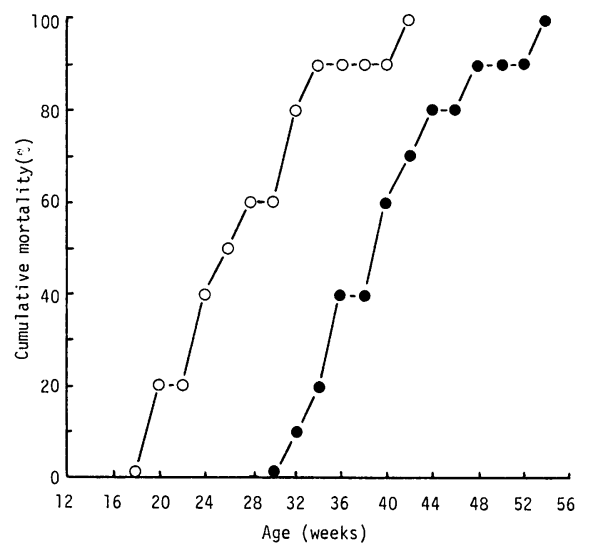

Fig. 1. The cumulative mortality rate of 2month-old female AKR/J mice treated with LC 9018. Mice $(n=10)$ received ip LC 9018 $(0.4 \mathrm{mg})$ twice weekly for 8 weeks, and the number of survivors were recorded once weekly. None (O), LC 9018

and experimental groups were $29.2 \pm 2.0$ and $41.6 \pm 2.5$ weeks $(p<0.01)$, respectively. Body weight of mice in the control group gradually increased and reached a maximum 18 to 20 weeks after birth $(20.0 \pm 0.8 \mathrm{~g})$, then decreased thereafter. In contrast, body weight of the LC 9018-administered mice reached a maximum 16 weeks after birth, retained maximal level for 8 weeks $(22.6 \pm 0.6 \mathrm{~g})$, and decreased gradually thereafter (data not shown).

Figure 2 shows the cumulative mortality rates of LC 9018-administered and control 3-month-old female AKR mice. Death in the experimental group first occurred at 32 weeks after birth, whereas death in the control first occurred at 20 weeks after birth. The mean survival age of mice in the experimental group (39.8 \pm 1.9 weeks) was significantly $(p<0.01)$ longer than that in the control group $(28.1 \pm 2.1$ weeks). The increase in body weight in the experimental animals was more marked than in the controls throughout the entire experiment (data not shown).

Figure 3 shows the cumulative mortality rates of LC 9018-administered and control 5-month-old female AKR mice. There were no significant differences in either the minimum time up to the death or the mean

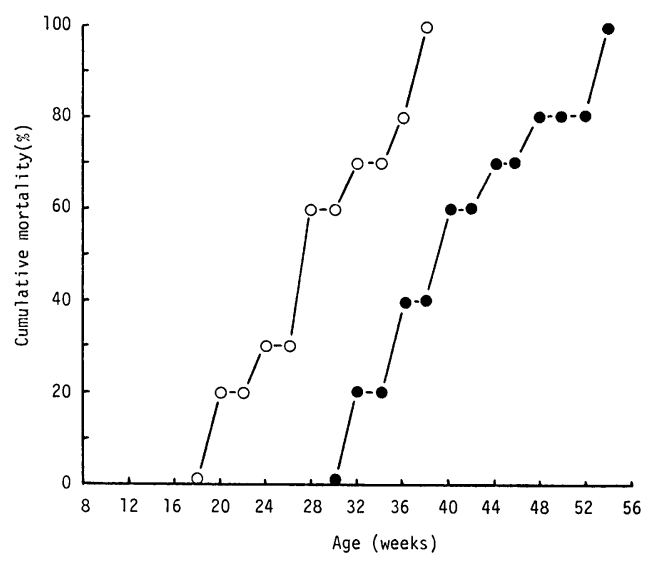

Fig. 2. The cumulative mortality rate of 3month-old female AKR/J mice treated with LG 9018. The protocol was the same as decribed in the legend for Fig. 1. None (O), LC 9018 (O).

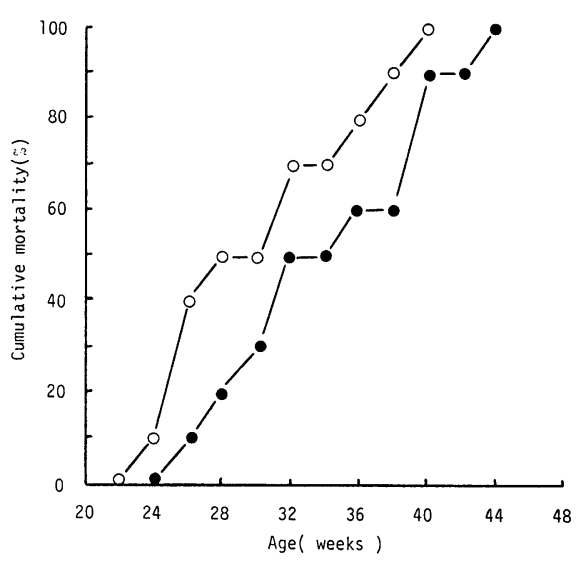

Fig. 3. The cumulative mortality rate of 5month-old female AKR/J mice treated with LC 9018. The protocol was the same as described in the legend for Fig. 1. None (O), LC 9018 (@).

survival age between control and experimental groups. There were also no differences in body weight between them (data not shown).

The thymus of LC 9018-administered and control 2-month-old AKR female mice was weighed 12 weeks after the last injection of LC 9018. As shown in Fig. 4, the enlargement of the thymus was significantly inhibited by the administration of the agent $(p<0.01)$. 


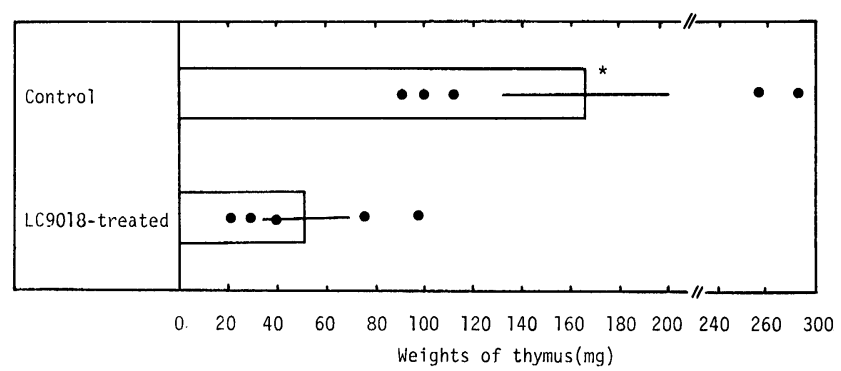

Fig. 4. Weights of thymus from 2-month-old female $\mathrm{AKR} / \mathrm{J}$ mice twelve weeks after the treatment with LC 9018. Mice $(n=5)$ given ip LC 9018 ( $0.4 \mathrm{mg} /$ week) for 8 weeks were decapitated 12 weeks after the last injection. Thymus was removed and weighed. $P$ values were calculated by Student's $t$-test. Bars: mean \pm S.E., $* p<0.01$ against control group.

Aoki et al. (1) reported that OK-432 (preparation of attenuated Streptococcus pyogenes $\mathrm{Su}$ treated with penicillin) suppressed the replication of MuLVs and delayed the incidence of spontaneous leukemia in 2- and 5-month-old AKR/J mice. In the present study, we found that the intraperitoneal administration of LC 9018 induced prolongation of the mean survival age and inhibition of thymus-enlargement in 2- and 3-monthold female $\mathrm{AKR} / \mathrm{J}$ mice, as compared with findings in the control mice, whereas these events did not occur in 5-month-old female mice administered LC 9018. The difference in the effectiveness of LC 9018 between 2to 3-month-old and 5-month-old mice may be due to the difference in the stage of spontaneous leukemogenesis. O'Donnell et al. (8) reported that representation of spontaneous leukemogenesis in AKR mice is distinguished by three stages: stage I, the infection of recombinant MuLVs to thymocytes, with no changes in the expression of several alloantigens on the major thymocyte subpopulations; stage II, the production of MuLVs and transformation of infected thymocytes; and stage III, the outgrowth of fully transformed cells and the appearance of thymus enlargement. Thus, the LC 9018-induced prolongation of the life span of 2- to 3-month-old AKR mice is assumed to be related to suppression of the production of MuLVs or of propagation of transformed thymic cells by activated effector cells such as macrophages, natural killer cells, and antibody-dependent killer cells $(4,5$,
13). It was reported that titers of ecotropic (XC cell focus-inducing) MuLVs per gram of thymus from young (1- to 2-month-old) $\mathrm{AKR} / \mathrm{J}$ mice were significantly lower than those of the thymus from adult (5- to 6month-old) animals, and that the rate of thymus weight to body weight in the former was smaller than that in the latter (11). Therefore, LC 9018-induced inhibition of the enlargement of thymus may be due to the suppression of production of MuLVs and/ or of growth of transformed thymic cells. In contrast, the administration of LC 9018 to 5-month-old female $\mathrm{AKR} / \mathrm{J}$ mice was ineffective. The result is assumed to be related to the decrease in the activated various effector cells or to the transformation of them by MuLVs, because animals in this period already had a severe leukemia, including the outgrowth of fully transformed thymic cells and the enlargement of thymus and lymph node $(8,11)$.

On the basis of the observations presented here, it was found that an intraperitoneal administration of LC 9018 effectively delays the incidence of spontaneous thymic lymphoma in young AKR mice, but not in adult mice.

\section{References}

(1) Aoki, T., J.P. Kvedar, Jr., V.W. Hollis, and G.S. Bushar. 1976. Streptococcus pyogenes preparation, OK-432: immunoprophylactic and immunotherapeutic effects on the incidence of spontaneous leukemia in AKR mice. J. Natl. Cancer Inst. 
56: 687-690.

(2) Herr, W. 1984. Necleotide sequence of AKR murine leukemia virus. J. Virol. 49: 471-478.

(3) Kato, I., S. Kobayashi, T. Yokokura, and M. Mutai. 1981. Antitumor activity by Lactobacillus casei in mice. Gann 72: 517-523.

(4) Kato, I., T. Yokokura, and M. Mutai. 1983. Macrophage activation by Lactobacillus casei in mice. Microbiol. Immunol. 27: 611-618.

(5) Kato, I., T. Yokokura, and M. Mutai. 1984. Augmentation of mouse natural killer cell activity by Lactobacillus casei and its surface antigens. Microbiol. Immunol. 28: 209-217.

(6) Li, Y., C.A. Holland, J.W. Hartley, and N. Hopkins. 1984. Viral integration near $c-m y c$ in 10-20\% of MCF 247-induced AKR lymphomas. Proc. Natl. Acad. Sci. U.S.A. 81 : 6808-6811.

(7) Lynch, C.J. 1954. The RIL strain of mice: its relation to leukemic AK strain and AKR substrain. J. Natl. Cancer Inst. 15: 161-176.

(8) O'Donnell, P.V., R. Woller, and A. Chu. 1984. Stages in development of mink cell focusinducing (MCF) virus-accelerated leukemia in
AKR mice. J. Exp. Med. 160: 914-934.

(9) Saito, H., T. Watanabe, and Y. Horikawa. 1981. Enhanced resistance to opportunistic infection in mice pretreated with Lactobacillus. Med. Biol. 102: 309-314.

(10) Sato, K. 1984. Enhancement of host resistance against Listeria infection by Lactobacillus casei: role of macrophages. Infect. Immun. 44: 445-451.

(11) Watanabe, T., and K. Nakakuki. 1977. A positive difference in nature of envelopes of thymusand uterus-derived leukemia viruses of AKR mice. Can. J. Microbiol. 23: 354-357.

(12) Watanabe, T., and H. Saito. 1986. Protection of mice against herpes simplex virus infection by Lactobacillus casei preparation (LC 9018) in combination with inactivated viral antigen. Microbiol. Immunol. 30: 111-122.

(13) Yasutake, N., M. Ohwaki, T. Yokokura, and M. Mutai. 1984. Comparison of antitumor activity of Lactobacillus case $i$ with other bacterial immunopotentiators. Med. Microbiol. Immunol, 173: 113-125. 